\title{
Quantifying micro-niche behaviour in sediments:- Precise and accurate measurements of DGT gels by laser ablation high resolution laser ablation ICPMS
}

\author{
D.G. PEARSON ${ }^{1}$, G.M. NOWELl1, A. WiderLUnD² \\ AND W. DAVISON ${ }^{2}$ \\ ${ }^{1}$ Department of Earth Sciences, University of Durham, UK; \\ ${ }^{2}$ Lancaster University, Department of Environmental \\ Science, Lancaster, LA1 4YQ, UK;
}

New developments in micron-scale measurements of trace metals and sulphur contents within sediments by DGT (diffusive gradients in thin-films) have revealed micro-niche structures. The origin of micro-niches is unclear and it is important to understand the relative roles played by inorganic and biological processes in the localised remobilisation of metals. We have undertaken a S and Fe isotope study of micro-niches, to better understand their mechanisms of formation. With their inbuilt pre-concentration, element selection and simple matrix, DGTs are an ideal medium for laser ablation ICP-MS. In contrast to analysis of mineral phases, fully matrix-matched standards can be easily made. We have initially concentrated on the measurement of $\mathrm{S}$ isotopes and will also present experiments where Fe isotopes have been measured in DGT gels. S and Fe isotopes were measured on a Neptune at mass resolutions of $>9000$. A NewWave UP-213nm laser was used with $\mathrm{Ar}$ as the carrier gas. Instrumental mass bias (IMB) for $\mathrm{S}$ was corrected for using sample-standard bracketing. Ablation experiments on two S DGT gel standards, $10 \mathrm{~Hz}, 40 \%$ power, $170 \mu \mathrm{m}$ wide line, scan speed $70 \mu \mathrm{ms}-1$, show in-run $\delta^{34} \mathrm{~S}$ precisions for DGT gels with $2.1 \mu \mathrm{mol} / \mathrm{cm}^{2} \mathrm{~S}$ ( $3 \mathrm{~V}$ of ${ }^{32} \mathrm{~S}$ ) of $<0.2 \%$ o (2SE). There is no observed correlation between $\delta^{34} \mathrm{~S}$ and ${ }^{32} \mathrm{~S}$ intensity. Reproducibility on $\delta^{34} \mathrm{~S}$ for individual analytical sessions (4-8 standards) is better than $0.3 \%$ while the reproducibility over 4 sessions is $0.19 \%$. Further tests to mimic ablation over micro-niches (continuous ablation across a gel with two isotopic compositions) show similar internal precisions and only slightly worse reproducibility $-0.48 \%$ o $(2 \mathrm{SD} ; \mathrm{n}=6)$. The accuracy of the LA $\delta^{34} \mathrm{~S}$ measurements on a $\mathrm{BaSO}_{4}$ gel relative to the reference value of the starting $\mathrm{BaSO}_{4}$, bracketed by a AgI gel of known isotopic composition, is better than $1 \%$, showing negligible $\mathrm{S}$ isotopic fractionation during gel deployment. $\delta^{34} \mathrm{~S}$ variations in micro-niches can be easily resolved at the $1 \%$ level and $100 \mu \mathrm{m}$ scale whereas variations in natural micro-niches are expected to be $10 \%$ or more. Fe measurements, using external normalisation to a ${ }^{60} \mathrm{Ni} /{ }^{61} \mathrm{Ni}$ ratio and an exponential law to correct for IMB, suggest internal precisions and external reproducibility of $<0.15 \%$ o (2SE) and $0.07 \%$ o (2SD) should be possible. 